\title{
Efecto del programa Aprender a Convivir en la competencia social y en los problemas de conducta del alumnado de 3 años
}

\author{
Ana Justicia-Arráez*, Carmen Pichardo y Fernando Justicia.
}

Universidad de Granada (España).

Resumen: La competencia social facilita el ajuste social y escolar del individuo siendo un importante factor de protección frente a la aparición de problemas de conducta. La adquisición de habilidades relacionadas con esta competencia depende en gran medida del ambiente y la estimulación temprana, cobrando gran importancia los programas de intervención educativa. El objetivo de este estudio consiste en analizar los efectos de un programa de intervención temprana (Aprender a Convivir) dirigido al desarrollo de la competencia social del alumnado de 3 años. Para valorar el impacto del programa se tomaron medidas pretest y postest entre los participantes y se realizó la comparación entre el grupo control $(n=151)$ y el grupo experimental $(\mathrm{n}=162)$ a través de un ANOVA mixto de medidas repetidas. Por otro lado, se llevó a cabo un ANCOVA para eliminar las diferencias pretest entre los grupos en el caso de que fuesen significativas. Los resultados de la investigación señalan una mejora del grupo experimental con respecto al grupo control en todas las variables de competencia social (cooperación $d=0.32$; interacción $d=0.65$; e independencia social $d=0.35)$ y de problemas de conducta (interiorizados $d=0.63$; y exteriorizados $d=0.57$ ) analizadas. También se hallaron diferencias de sexo en cada una de las variables.

Palabras clave: programa Aprender a Convivir; competencia social; educación infantil, problemas de conducta; prevención universal.

\section{Introducción}

La competencia social ha sido considerada por ciertos autores como un constructo multidimensional, pues su desarrollo implica la activación de diferentes procesos internos en el individuo (cognitivos, afectivos, conductuales), en una etapa evolutiva determinada y dentro de un contexto social específico (Rose-Krasnor, 1997; Vaughn et al., 2009; Waters y Sroufe, 1983). Partiendo de esta concepción compleja de la competencia social, Merrell (2002) sostiene que este constructo está compuesto por comportamientos prosociales, adaptativos y positivos, relacionados entre sí. Centrándonos en la etapa infantil este autor diferencia tres dimensiones dentro de la competencia social: cooperación, interacción e independencia. Dentro de la cooperación social se incluyen destrezas importantes relacionadas con el seguimiento de instrucciones y normas, conductas prosociales (cooperar, ayudar, etc.) y habilidades de auto-control. En la dimensión de interacción social se incluyen comportamientos relacionados con el mantenimiento de relaciones (ya sea con los adultos o entre iguales) que influyen en el grado de percepción y aceptación social que los demás tienen de uno mismo. Por último, la destreza de independencia social engloba conductas como aceptar la separación temporal de los adultos, mostrar confianza en los demás y cierto grado de autonomía.

La infancia es un periodo crucial en el desarrollo social y emocional de la persona constituyéndose como una etapa

* Dirección para correspondencia [Correspondence address]:

Ana Justicia Arráez. Dpto. Psicología Evolutiva y de la Educación. Campus de Cartuja s/n, 18071, Granada (España).E-mail: anajus@ugr.es
Title: Effect of the "Aprender a Convivir" program on social competence and behavioral problems in three-year-old children.

Abstract: Social competence facilitates social and school adjustment of the individual and has emerged as one of the most important protective factor against the development of behavioral problems. Acquiring skills related to this competence depends largely on the environment and early stimulation, gaining importance the educational intervention programs. The aim of this study was to analyze the effects of an early intervention program (Aprender a Convivir) directed towards social competence development of three-year old children. Pretest and postest measures were taken among the participants and the comparison was performed between the control group $(n=151)$ and the experimental group $(n=162)$ through a repeated-measures mixed ANOVA. On the other hand an ANCOVA was carried out in order to eliminate the pretest differences between groups in the case that would be significant. The results indicate a significant improvement in the experimental group compared with the control group in all the social competence (cooperation $d=0.32$; interaction $d=0.65$; and social independence $d=0.35$ ) and conduct problem (internalizing $d=0.63$; and externalizing $d=0.57$ ) variables analyzed. Sex differences were also found.

Key words: Aprender a Convivir program; social competence; preschool education; conduct problem; universal prevention.

donde la adquisición de competencia social es básica. Es necesario incidir sobre estos aspectos del desarrollo pues el aprendizaje de habilidades socio-emocionales depende del ambiente y la estimulación temprana (Joseph y Strain, 2003). La promoción de la competencia social en la infancia es una tarea educativa primordial, no solo por su contribución al desarrollo social y emocional de la persona, también porque este constructo mantiene una relación positiva con variables como el rendimiento escolar (Bradshaw, Bottiani, Osher y Sugai, 2014; Del Prette, Del Prette, De Oliveira, Gresham y Vance, 2012; Inglés et al., 2009), o la inteligencia emocional (Eisenberg, 2000); y una relación negativa con variables como los problemas de conducta (Alba, 2013; Daly, Nicholls, Aggarwal y Sander, 2014; Dodge, Godwin y The Conduct Problems Prevention Research Group, 2013; Justicia-Arráez, 2014). Según estos estudios, una persona con un nivel de competencia social elevado suele tener un mayor rendimiento escolar, domina mejor sus emociones y resuelve adecuadamente sus problemas interpersonales. En esta línea, Zins, Weissberg, Wang y Walberg (2004) señalan que los niños/as que reciben una formación socio-emocional están más conectados con la escuela, con los compañeros de clase, están más motivados para aprender, manifiestan comportamientos adecuados, tienen menor riesgo de presentar problemas de conducta y suelen alcanzar mayores logros académicos. Es por ello que autores como Reynolds et al. (2007) resaltan en su investigación la importancia de llevar a cabo estrategias educativas entre los 3 y los 9 años ya que así se contribuye positivamente a la salud mental y al bienestar personal del sujeto. Igualmente, las intervenciones tempranas suponen 
beneficios públicos y sociales, contribuyendo al bienestar económico del estado (Reynolds y Temple, 2008, 2012).

La consideración de la competencia social como un factor de protección para el desarrollo saludable y el bienestar del individuo (Ferrer-Wreder, 2014) ha provocado la aparición de programas de intervención en el ámbito educativo. Algunos ejemplos de programas dirigidos a la población infantil que trabajan la competencia social son el Programa de Asertividad y Habilidades sociales (PAHS), (Monjas, 2011); el programa Aprender a Convivir (Alba, Justicia-Arráez, Pichardo y Justicia, 2013); el Al's Pals (Cooper, Goodfellow, Muhlheim, Paske, y Pearson, 2003); el Strong Start Pre-K (Gunter, Caldarella, Korth y Young, 2012); o el PATHS Preschool: Promoting Alternative Thinking Strategies (Domitrovich, Greenberg, Kusche y Cortes 2004) (para más información relacionada con programas que trabajan la competencia social ver Alba et al., 2013). Estos programas de intervención han resultado muy positivos para la mejora de la competencia social del alumnado. En el ámbito español, los resultados obtenidos con el Programa de Enseñanza de Habilidades de Interacción Social- PEHIS (Monjas, 2012), muestran un incremento de las habilidades sociales de los participantes (Monjas y González, 1998). También se hallaron resultados positivos con alumnado de 4 años de edad tras la implementación del programa Aprender a Convivir (Benítez, Fernández, Justicia, Fernández y Justicia-Arráez, 2011a), dado que los alumnos pertenecientes al grupo experimental mejoraron de forma significativa con respecto a los del control en competencia social. En el ámbito internacional también se han obtenido resultados positivos tras la aplicación de programas de intervención educativa en población infantil, los cuales provocaron una mejora significativa en el comportamiento prosocial del alumnado (Kramer, Caldarella, Christensen y Shatzer, 2010; Domitrovich et al., 2007; Moraru, Stoica, Tomuletiu, Filpisan, 2011; Ştefan y Miclea, 2013).

Como se ha mencionado anteriormente, existe una relación negativa entre la competencia social y los problemas de conducta. Este tipo de problemas son dificultades que presenta el individuo ante determinadas circunstancias manifestándose mediante un comportamiento inadaptado. El estudio de los problemas de conducta focaliza su atención en dos tipos de manifestaciones: interiorización de problemas, esto es, conductas que el sujeto dirige hacia sí mismo, manifestándose en problemas como la ansiedad, la depresión o la timidez; y exteriorización de problemas, entendidos como conductas que se manifiestan hacia fuera o dirigidas hacia los otros, como pueden ser las conductas agresivas o los problemas de atención. Durante la infancia suele ser común la aparición de estos problemas, pero si perduran en el tiempo pueden derivar en un patrón de comportamiento más complejo, habiendo sido señalados como indicadores del comportamiento antisocial temprano (Bergman, Andershed y Andershed, 2009; Calkins y Keane, 2009).

Volviendo a la relación entre las variables, diversos estudios han mostrado que la falta de competencia social influye en el desarrollo de problemas de tipo interiorizado y exterio- rizado (Bornstein, Hahn y Haynes, 2010; Burt, Obradović, Long y Masten, 2008; Cole, Martin, Power y Truglio, 1996; Mesman, Bongers y Koot, 2001). Investigaciones como la de Bornstein et al. (2010) han analizado la relación entre estas variables en niños de 4 años de edad y los resultados muestran cómo ambas dimensiones -competencia social y problemas de conducta- se relacionan de forma negativa, es decir, un individuo con mayor nivel de competencia social es probable que manifieste menores problemas de conducta, y viceversa. Además, es importante mencionar que esta relación negativa parece ser estable a través del tiempo (Obradović, Burt y Masten, 2009; Sørlie, Hagen y Ogden, 2008), por lo que la competencia social adquiere gran relevancia como factor de protección frente a la aparición de problemas de conducta.

En este sentido, si no se realiza una intervención temprana o preventiva sobre problemas de conducta como la agresividad o la conducta oposicionista-desafiante, estas conductas pueden cristalizarse en torno a los 8 años de edad (Eron, 1990) o convertirse en una escalada hacia otras conductas como bajo rendimiento académico, absentismo escolar, abuso de sustancias tóxicas, delincuencia o violencia (Snyder, 2001). El hecho de iniciar una intervención con sujetos de temprana edad se debe a que la estabilidad de los problemas de conducta es más alta después de los 4 años y tiende a aumentar con la edad (Hemphill, 1996). A partir de los 3-4 años es cuando la frecuencia y complejidad de las interacciones sociales aumenta vertiginosamente por lo que los años preescolares son una gran oportunidad para prevenir el posible desarrollo de problemas de conducta (Tremblay, 2007).

Es una realidad que sin una intervención temprana efectiva los problemas de conducta, sociales y emocionales tienden a agravarse (Daly et al., 2014). Sin embargo, todavía son pocas las acciones de cierta amplitud y continuidad en el tiempo, siendo precisamente en la etapa de Educación Infantil donde menos esfuerzos se han invertido para diseñar e implementar programas de prevención de problemas de conducta (Justicia, Benítez, Fernández, Fernández y Pichardo, 2008). No obstante, existen algunos programas que trabajan en esta línea y que han encontrado resultados positivos. En el caso del programa Early Impact Program, los niños y niñas del grupo experimental disminuyeron los niveles de exteriorización e interiorización de problemas (Larmar, Dadde y Shochet, 2006). Igualmente, el programa Project Primar aporta resultados similares pues comportamientos de tipo antisocial se reducen considerablemente en los participantes del grupo experimental (Koglin y Petermann, 2011; Pettermann y Natzke, 2008).

Además de los argumentos aportados, la intervención dirigida a la mejora de la competencia social y a la reducción de problemas de conducta se debe iniciar desde edades tempranas ya que puede ayudar a minimizar las diferencias de género existentes en este tipo de comportamientos. Investigaciones llevadas a cabo con niños y niñas de preescolar han puesto de manifiesto la influencia del género tanto en diver- 
sos comportamientos sociales como en problemas de conducta. En habilidades relacionadas con la competencia social, las niñas tienden a ser más empáticas (Schulte-Ruther, Markowitsch, Shah, Fink, y Piefke, 2008) y más conciliadoras que los niños, que por el contrario, persiguen metas sociales en las que ejercen un rol más dominante (Walker, 2005). Del mismo modo, otros estudios recientes han puesto de manifiesto una mayor habilidad de las niñas para reconocer e interpretar las emociones (Fabes y Eisenberg, 1998; Öztürk Samur, 2014; Ştefan y Miclea, 2013). Por otro lado, durante los años preescolares niños y niñas suelen mostrar niveles similares de problemas de conducta (Keenan y Shaw, 1994). Sin embargo, autores como Offord et al. (1987) sostienen que mientras que la manifestación de problemas exteriorizados aumenta en los niños a partir de los 4 años, la interiorización sigue siendo similar en ambos sexos a esta edad. Concretamente en dimensiones como la timidez, revisiones recientes señalan que no existen diferencias de sexo durante la infancia temprana (Doey, Coplan y Kingsbury, 2013). Incidir directamente, mediante programas de intervención, sobre aspectos relacionados con el comportamiento prosocial, los sentimientos, las habilidades de interacción social, la resolución de problemas, entre otros, puede ayudar a frenar la aparición de diferencias de género, máxime si se trabaja desde la coeducación, por lo que conviene seguir profundizando sobre la variable del género para delimitar con claridad a qué edad se hacen manifiestas las diferencias entre niños y niñas para así poder facilitar una intervención adecuada.

A fin de aportar mayor evidencia empírica acerca del impacto que supone la promoción de las intervenciones tempranas que desarrollan la competencia social, el objetivo fundamental de este estudio consiste en analizar el efecto del programa de prevención universal Aprender a Convivir implementado con alumnado de 3 años de edad. En primer lugar, se analizará el efecto del programa partiendo de la consideración de que el alumnado que participa en dicho programa, en comparación con el grupo control, mejorará de manera significativa su competencia social (H1) y disminuirá sus puntuaciones en las variables de problemas de conducta (H2). Por otro lado, en la presente investigación también se analizará la influencia del sexo en las variables analizadas. Dadas las diferencias que se advierten entre niños y niñas tanto en competencia social como en problemas de conducta, se espera que las niñas tengan mejores niveles de competencia social $(\mathrm{H} 3)$ y presenten menos problemas de conducta exteriorizados $(\mathrm{H} 4)$ que los niños.

\section{Método}

\section{Participantes}

En el estudio participaron 313 alumnos/as de 3 años procedentes de ocho centros educativos concertados de Granada capital, situados en un entorno socioeconómico medio. Para la selección de los centros se utilizó un muestreo de tipo probabilístico incidental. La asignación de los centros participantes al grupo experimental o al grupo control se hizo aleatoriamente antes de realizar la evaluación del alumnado. Tras haber obtenido el consentimiento de las familias, todas las clases de 3 años de cada uno de los centros fueron incluidas en la investigación.

En cuanto a la distribución de los participantes, según el grupo y el sexo, indicar que el grupo experimental estuvo formado por 162 sujetos (el $46.9 \%$ eran niños y el 53.1\% niñas), procedentes de cuatro centros educativos. Por otra parte, el grupo control estuvo conformado por 151 sujetos (un $49.7 \%$ niños y un $50.3 \%$ niñas), procedentes de otros cuatro centros educativos de similares características a los centros experimentales.

\section{Instrumentos}

Para evaluar las variables de competencia social y de problemas de conducta se utilizaron los siguientes instrumentos:

Preschool and Kindergarten Behavior Scale for Teachers and Caregivers, PKBS-2 (Merrell 2002). Para la evaluación de la competencia social del alumnado de 3 años se utilizó la versión traducida y adaptada al español denominada Escala de Observación en Educación Infantil -EOEI- (Benítez, Pichardo, García, Fernández, Justicia y Fernández, 2011b; Fernández et al., 2010). La escala de competencia social, con 34 ítems, se puede aplicar a niños entre los 3 y los 6 años de edad, tanto por docentes como por otros cuidadores. Se trata de una escala Likert con valores que oscilan entre 0 (nunca) y 3 (con frecuencia) y está conformada por tres subescalas: cooperación social (12 ítems), interacción social (11 ítems) e independencia social (11 ítems). La estructura de la escala fue confirmada en población española por Benítez et al., (2011b), mediante un análisis factorial confirmatorio con valores de ajuste y de fiabilidad adecuados. Los coeficientes de consistencia interna a partir de la muestra del presente estudio también han sido adecuados: escala de competencia social $\alpha=.94$, y para las subescalas de cooperación social $\alpha=.90$, interacción social $\alpha=.91$ e independencia social $\alpha=89$.

Child Behavior Checklist- Teacher Report Form (CBCL-TRF) de Achenbach y Rescorla (2000). Para medir los problemas de conducta se utilizó este instrumento en su versión TRF para maestros/as y cuidadores. Se trata de una escala tipo Likert con valores que oscilan entre 0 (no es cierto) y 2 (a menudo, bastante). El cuestionario se divide en dos subescalas: a) interiorización de problemas, conformada por las variables de reactividad emocional, ansiedad-depresión, quejas somáticas y timidez; y b) exteriorización de problemas, compuesta por las variables de problemas de atención y comportamiento agresivo. La escala original presenta índices de fiabilidad y validez adecuados (Achenbach y Rescorla, 2000). Los coeficientes de consistencia interna a partir de la muestra del presente estudio han sido adecuados, siendo para la escala total $\alpha=.93$, y para la subescala de interiorización de problemas $\alpha=.89$, y de exteriorización de problemas $\alpha=.93$. 


\section{Procedimiento}

Después de haber obtenido el consentimiento de colaboración en los centros experimentales y controles, todas las maestras de las clases que participaban en el estudio cumplimentaron los cuestionarios tras un periodo de observación durante el primer trimestre (pre-evaluación) del curso escolar. En la fase de observación, a las maestras se les facilitó un registro de observación para el aula y otro para el recreo, con el fin de que se centraran en analizar la frecuencia de las conductas concretas que posteriormente iban a evaluar. Durante el segundo trimestre y parte del tercero, maestras e investigadoras implementaron el programa Aprender a Convivir en los centros experimentales. La duración del programa fue de doce semanas a razón de dos sesiones semanales. En el grupo control no se hizo intervención.

El programa Aprender a Convivir (ver Alba et al., 2013; Justicia et al., 2008) utilizado para esta investigación fue el específico para alumnos de tres años. Dicho programa se divide en cuatro bloques temáticos: Las normas y su cumplimiento, Sentimientos y emociones, Habilidades de comunicación y Ayuda y cooperación. De manera transversal, en cada uno de los bloques se trabaja sobre la resolución de problemas. Cada bloque está compuesto por seis sesiones de trabajo agrupadas en tres unidades temáticas. La estructura de la sesión y el sistema de trabajo comienza con una actuación de marionetas que presentan los objetivos fundamentales a trabajar ese día, además de plantear situaciones problemáticas a las que el alumnado debe dar solución. Posteriormente, se realiza una actividad concreta (en gran grupo, pequeño grupo o de forma individual) para enfatizar sobre el contenido propio de la sesión.

Tras el periodo de implementación del programa, las maestras iniciaron un nuevo periodo de observación hasta el final del curso, momento en que cumplimentaron los cuestionarios (post-evaluación).

\section{Análisis estadístico}

Se realizaron análisis preliminares para explorar, dentro de cada grupo, las puntuaciones medias y desviaciones típicas de cada una de las variables. Posteriormente, se realizó un ANOVA mixto de medidas repetidas $2 \mathrm{x}(2 \mathrm{x} 2)$, incluyendo como factor intra-grupo el momento de la evaluación (pretest-postest) y como factores entre-grupos la condición del grupo (experimental-control) y el sexo (niño-niña). Por otro lado, se llevó a cabo un análisis de la covarianza univariante (ANCOVA) para eliminar las diferencias pretest entre los grupos en el caso de que fuesen significativas. Finalmente, fueron calculados los tamaños del efecto (índice $d$ o diferencia media tipificada), propuesto por Cohen (1988), los cuales permiten cuantificar la magnitud de las diferencias encontradas, aportando más información sobre el tamaño del efecto del programa en las variables dependientes analizadas en el estudio. Este mismo autor establece efectos pequeños $(.20 \leq d \leq .49)$, moderados $(.50 \leq d \leq .79)$ y grandes $(d \geq .80)$. Los datos se analizaron con el programa estadístico IBM SPSS Statistics 20.0.

\section{Resultados}

\section{Competencia social}

En los análisis preliminares se calcularon los estadísticos descriptivos para las variables de competencia social (tabla 1), incluyendo las medias y desviaciones típicas de los niños y niñas de ambos grupos (experimental-control) en los dos momentos de evaluación (pretest-postest).

Los resultados obtenidos del ANOVA mixto de medidas repetidas indican para la variable de cooperación social la existencia de efectos principales del factor momento de evaluación $\left(F_{(1,295)}=95.64, p<.000\right)$, siendo mayores las puntuaciones de los participantes en el postest. También hubo efectos principales del factor sexo $\left(F_{(1,295)}=12.79, p<.000\right)$, siendo las niñas las que puntuaron más alto en esta variable. Por otro lado, el factor condición no mostró efectos principales $\left(F_{(1,295)}=2.18, p=.141\right)$, sin embargo, se hallaron efectos de interacción entre los factores momento de evaluación*condición $\left(F_{(1,295)}=8.23, p=.004\right)$. Los sujetos de ambos grupos partían de puntuaciones similares en la fase pre, pero en la fase post se encontraron diferencias estadísticamente significativas en favor del grupo experimental (Figura 1). El tamaño del efecto de las diferencias entre controles y experimentales en la fase postest, realizado a través del estadístico $\mathrm{d}$ de Cohen, mostró un efecto pequeño $(d=0.32)$.

Tabla 1. Medias y desviaciones típicas de los participantes en las variables de Competencia Social.

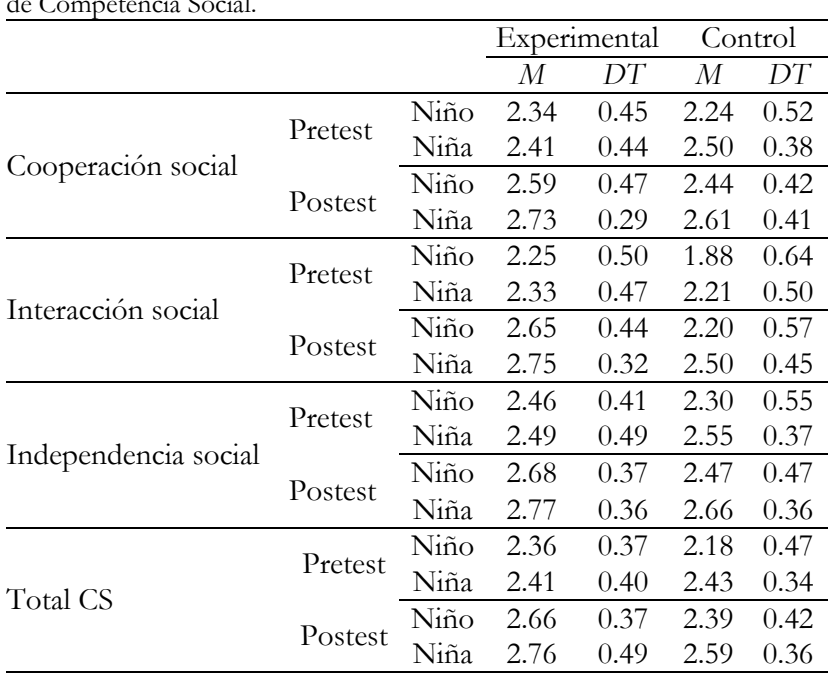

En el caso de la variable interacción social se encontraron efectos principales del factor momento de evaluación $\left(F_{(1,300)}=209.09, p<.000\right)$, siendo mayores las puntuaciones obtenidas en el postest. Además, hubo efectos principales del factor condición $\left(F_{(1,300)}=35.51, p<.000\right)$ a favor del grupo experimental, y del factor sexo $\left(F_{(1,300)}=15.93\right.$, $\left.p<.000\right)$, con 
puntuaciones más elevadas para las niñas. Por otra parte, se hallaron efectos de interacción entre los factores momento de evaluación*condición $\left(F_{(1,300)}=4.48, p=.035\right)$. En ambos grupos, los valores de esta variable aumentaron del pre al post, pero los sujetos del grupo experimental incrementaron más su puntuación que los del grupo control en el postest. Tal y como se puede observar en la Figura 2, a pesar de que el alumnado del grupo experimental partía de niveles más altos en el pretest, el ANCOVA $\left(F_{(1,301)}=216.45, p<.000\right)$ realizado muestra cómo las diferencias en el post siguen siendo significativas en favor del grupo experimental, con un tamaño del efecto moderado $(d=0.65)$.

En relación con la variable independencia social, también hubo efectos principales de los tres factores: momento de evaluación $\left(F_{(1,299)}=65.30, p<.000\right)$, condición $\left(F_{(1,299)}=5.28\right.$, $p=.022)$ y sexo $\left(F_{(1,299)}=10.64, p=.001\right)$. En el caso del factor momento de evaluación, las puntuaciones fueron más elevadas en el postest; en cuanto al factor condición, las diferencias fueron significativas en favor del grupo experimental pues alcanzó puntuaciones más elevadas que el grupo control; y en relación con el sexo, fueron las niñas las que obtuvieron puntuaciones más elevadas. Por último, se hallaron

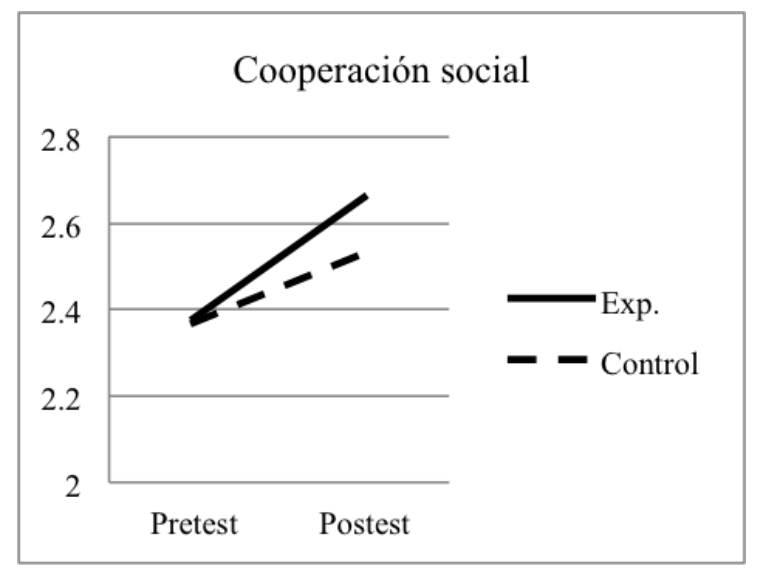

Figura 1. Efectos de interacción entre el momento de evaluación (pretest-postest) y la condición (experimental-control), en la variable cooperación social. efectos de interacción entre los factores momento de evaluación*condición $\left(F_{(1,299)}=4.55, p=.034\right)$, siendo los participantes del grupo experimental los que alcanzaron puntuaciones significativamente más elevadas que los del grupo control en la fase post, partiendo de puntuaciones similares en la fase pre (Figura 3). El tamaño del efecto al comparar los controles y experimentales en la fase postest es pequeño $(d=0.35)$.

Respecto a la variable total de competencia social, también se obtuvieron efectos principales de los factores momento de evaluación $\left(F_{(1,295)}=155.32, p<.000\right)$, condición $\left(F_{(1,295)}=14.42\right.$, $p<.000)$ y sexo $\left(F_{(1,295)}=14.25, p<.000\right)$. Con respecto al momento de evaluación, las puntuaciones fueron más elevadas en la fase post. En el caso del factor condición, se encontraron diferencias estadísticamente significativas en favor del grupo experimental. Respecto del sexo, hubo diferencias significativas que indicaban mayores puntuaciones de competencia social por parte de las niñas. Por otro lado, hubo efectos de interacción entre los factores momento de evaluación*condición $\left(\mathrm{F}_{(1,295)}=11.20, p=.001\right)$, que evidenciaron diferencias significativas en el postest a favor del grupo experimental, con un tamaño del efecto moderado, $d=0.54$ (Figura 4), diferencias que no estaban presentes en el pretest.

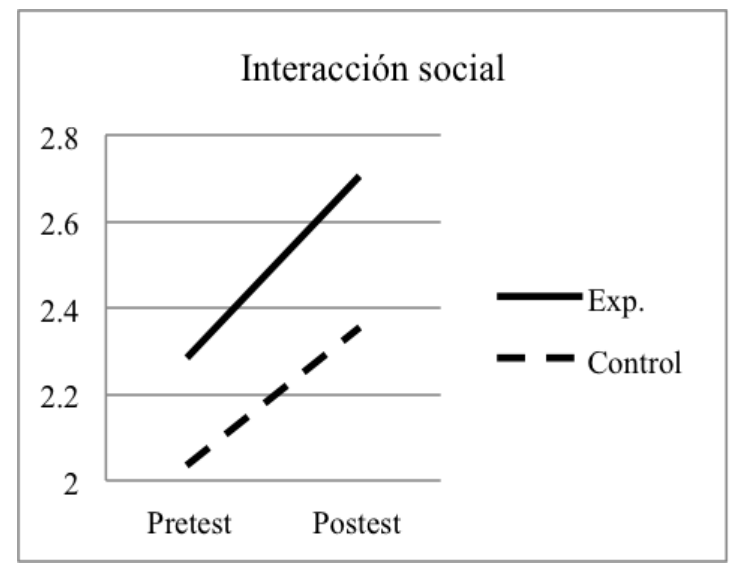

Figura 2. Efectos de interacción entre el momento de evaluación (pretest-postest) y la condición (experimental-control), en la variable interacción social. 


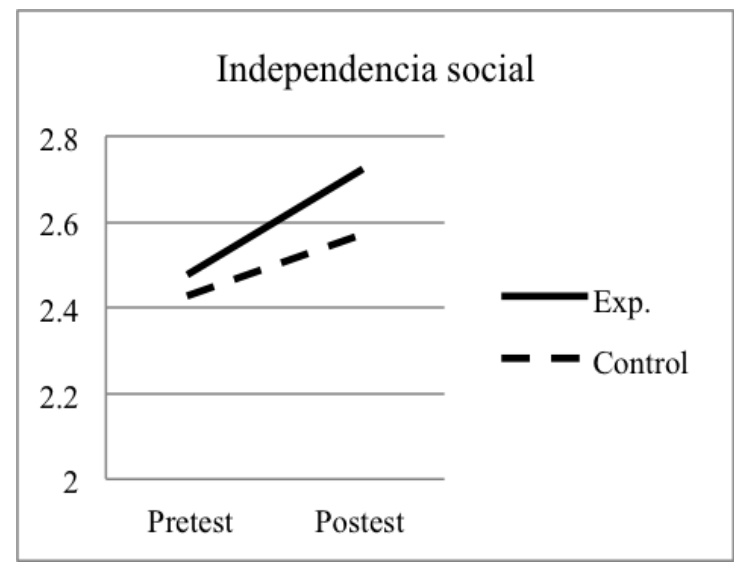

Figura 3. Efectos de interacción entre el momento de evaluación (pretest-postest) y la condición (experimental-control), en la variable de independencia social.

\section{Problemas de conducta}

Se calcularon los estadísticos descriptivos para las variables de problemas de conducta (tabla 2), incluyendo las medias y desviaciones típicas de los niños y niñas de ambos grupos (experimental-control) y en los dos momentos de evaluación (pretest-postest).

Los efectos principales de la variable interiorización de problemas -conformada por reactividad emocional, ansiedaddepresión, quejas somáticas y timidez-, fueron significativos para el factor momento de evaluación $\left(F_{(1,303)}=37.73\right.$, $p<.000)$, siendo en el postest donde las puntuaciones son más bajas; el factor condición $\left(F_{(1,303)}=25.62, p<.000\right)$, con diferencias entre ambos grupos en favor del grupo experimen-

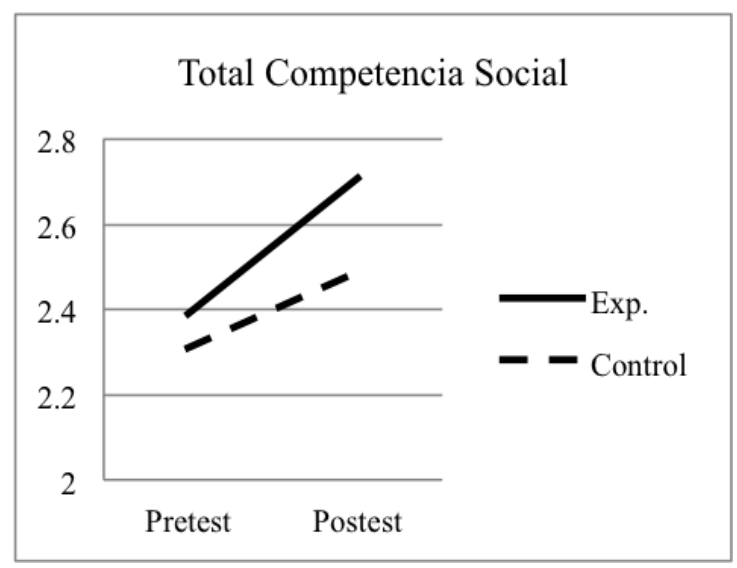

Figura 4. Efectos de interacción entre el momento de evaluación (pretest-postest) y la condición (experimental-control), en la variable total de competencia social.

tal; y el factor sexo $\left(F_{(1,303)}=8.69, p=.003\right)$, siendo los niños los que presentaron puntuaciones más elevadas. Por otro lado, se encontraron efectos de interacción entre momento de evaluación* condición $\left(F_{(1,303)}=13.09, p<.000\right)$, ya que se produjo un descenso mayor en las puntuaciones del grupo experimental del pretest al postest (Figura 5). A pesar de que el grupo experimental partía con puntuaciones significativamente más bajas que el grupo control, el ANCOVA $\left(F_{(1,304)}=253.55, p<.000\right)$ realizado indica que aun restando el efecto de la medida pretest, las diferencias siguen siendo significativas en la fase postest a favor del grupo experimental, con un tamaño del efecto moderado $(d=0.63)$. El grupo control reduce sus puntuaciones en el postest, pero no de forma significativa.

Tabla 2. Medias y desviaciones típicas de los participantes en las variables de Problemas de Conducta.

\begin{tabular}{|c|c|c|c|c|c|c|}
\hline & & & \multicolumn{2}{|c|}{ Experimental } & \multicolumn{2}{|c|}{ Control } \\
\hline & & & $M$ & $D T$ & $M$ & $D T$ \\
\hline \multirow{4}{*}{ Interiorización de problemas } & \multirow{2}{*}{ Pretest } & Niño & 0.62 & 0.72 & 1.07 & 1.16 \\
\hline & & Niña & 0.59 & 0.68 & 0.65 & 0.69 \\
\hline & \multirow{2}{*}{ Postest } & Niño & 0.33 & 0.40 & 0.92 & 0.91 \\
\hline & & Niña & 0.19 & 0.24 & 0.62 & 0.66 \\
\hline \multirow{4}{*}{ Exteriorización de problemas } & \multirow{2}{*}{ Pretest } & Niño & 0.61 & 0.64 & 0.80 & 0.63 \\
\hline & & Niña & 0.47 & 0.51 & 0.50 & 0.55 \\
\hline & \multirow{2}{*}{ Postest } & Niño & 0.37 & 0.46 & 0.81 & 0.62 \\
\hline & & Niña & 0.22 & 0.29 & 0.52 & 0.65 \\
\hline \multirow{4}{*}{ Total Problemas Conducta } & \multirow{2}{*}{ Pretest } & Niño & 1.16 & 0.90 & 1.87 & 1.51 \\
\hline & & Niña & 1.08 & 0.95 & 1.16 & 1.10 \\
\hline & \multirow{2}{*}{ Postest } & Niño & 0.69 & 0.73 & 1.73 & 1.30 \\
\hline & & Niña & 0.42 & 0.44 & 1.15 & 1.15 \\
\hline
\end{tabular}

En exteriorización de problemas, -conformada por problemas de atención y comportamiento agresivo-, los resultados fueron similares a lo observado en la interiorización de problemas, con efectos principales encontrados para el momento de evaluación $\left(F_{(1,299)}=18.94, p<.000\right)$, siendo las puntuaciones más bajas en el postest; condición $\left(F_{(1,299)}=16.68\right.$, $p<.000)$, con diferencias en favor del grupo experimental, que alcanzó puntuaciones significativamente más bajas que el grupo control; y sexo $\left(F_{(1,299)}=14.00, p<.000\right)$, habiendo diferencias en favor de las niñas, que obtienen puntuaciones más bajas. Por otro lado, hubo efectos de interacción entre el momento de evaluación*condición $\left(F_{(1,299)}=24.27, p<.001\right)$, 
que evidencian la existencia de diferencias significativas entre ambos grupos en la fase post, presentando un tamaño del efecto moderado $(d=0.57)$.. Los grupos experimental y control obtienen puntuaciones similares en exteorización de problemas en la fase pretest. Sin embargo, en la fase postest, el grupo experimental reduce significativamente sus puntuaciones, mientras que en el grupo control se mantienen (Figura 6).

Por último, en la variable total de problemas de conducta se encontraron efectos principales del momento de evaluación $\left(F_{(1,296)}=40.09, p<.000\right)$ siendo las puntuaciones más bajas en el postest. También hubo efecto principal del factor condición $\left(F_{(1,296)}=33.42, p<.000\right)$, ya que el grupo experimental obtuvo puntuaciones más bajas; y del factor sexo

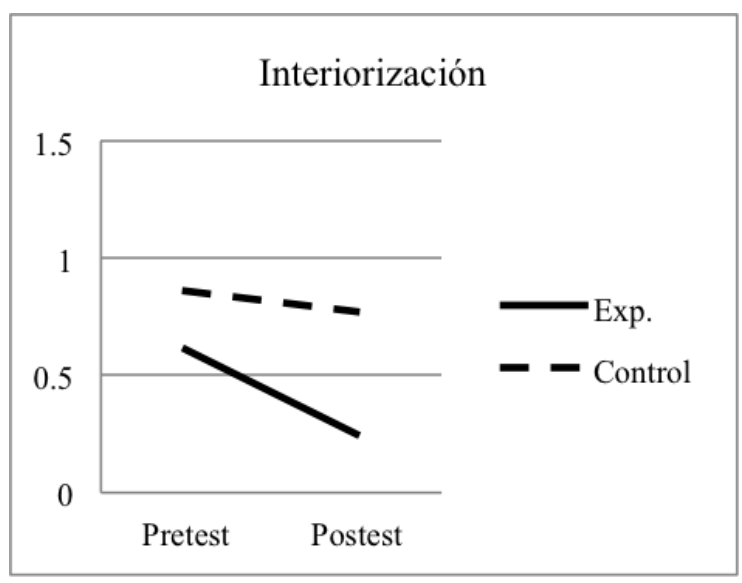

Figura 5. Efectos de interacción entre la evaluación (pretestpostest) y la condición (experimental-control), en la variable interiorización de problemas.
$\left(F_{(1,296)}=13.90, p<.000\right)$, siendo las niñas las que puntuaron más bajo. Además, se hallaron efectos de interacción para el momento de evaluación*condición $\left(F_{(1,296)}=23.46, p<.000\right)$. Como se observa en la figura 7 , en el grupo control las puntuaciones fueron similares del pretest al postest, sin embargo, en el grupo experimental las puntuaciones fueron significativamente inferiores en la fase postest. A pesar de que el alumnado del grupo experimental partía de niveles más bajos en la fase pretest, el ANCOVA $\left(F_{(1,297)}=254.17, p<.000\right)$ realizado muestra cómo las diferencias en la fase postest siguen siendo significativas y por tanto, más bajas que las del grupo control, con un tamaño del efecto moderado $(d=0.70)$.

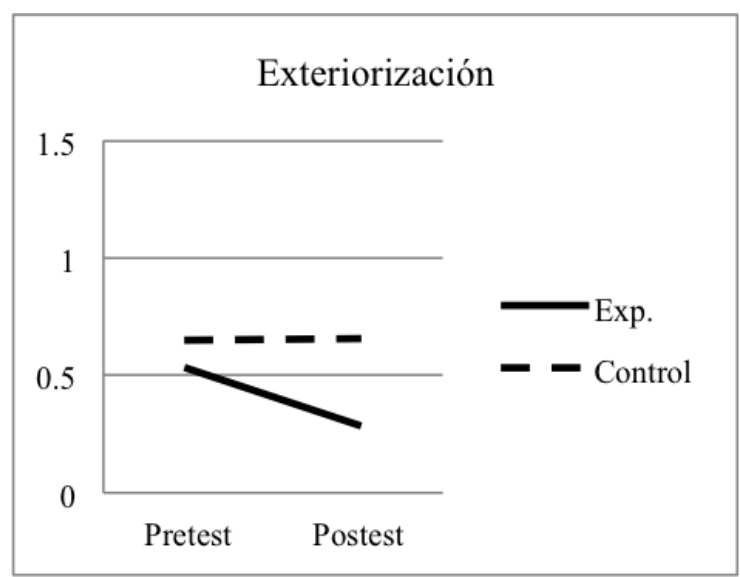

Figura 6. Efectos de interacción entre la evaluación (pretestpostest) y la condición (experimental-control), en la variable exteriorización de problemas.

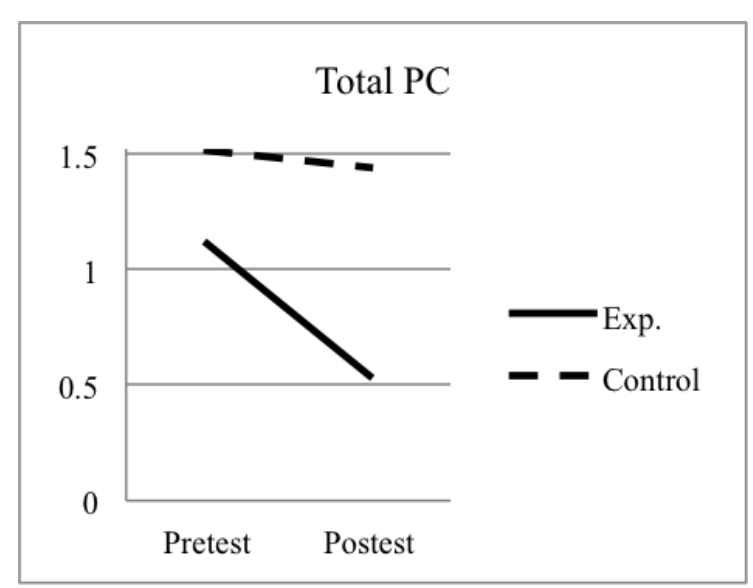

Figura 7. Efectos de interacción entre la evaluación (pretest-postest) y la condición (experimental-control), en la variable total de problemas de conducta.

\section{Discusión}

Los resultados obtenidos en el estudio acerca del efecto del programa Aprender a Convivir para Educación Infantil muestran que la intervención realizada contribuye a la mejora de la competencia social del alumnado de 3 años. Los efectos de la intervención fueron significativos para cada una de las variables analizadas pues los niños y niñas que participaron en la 
intervención mejoraron considerablemente tanto en competencia social como en problemas de conducta interiorizados y exteriorizados.

Atendiendo a los resultados obtenidos en competencia social, se confirma la hipótesis de partida (H1) pues el alumnado que ha participado en el programa, adquiere una mayor competencia social que el que no lo ha hecho. Los participantes del grupo experimental son más competentes socialmente, es decir, cooperan más, aumentan su capacidad de interacción con los otros y son más independientes. Ambos grupos mejoraron su nivel de competencia social, pero la condición experimental y el momento de evaluación han marcado claras diferencias entre los participantes, indicando los efectos de interacción que el alumnado del grupo experimental es el que ha obtenido mejores puntuaciones en la fase post. Ha sido en la capacidad para interaccionar con los demás donde el efecto del programa fue mayor.

Es lógica la mejora en ambos grupos ya que el sujeto está en pleno desarrollo e inicia a esta edad nuevas interacciones con sus iguales, consideradas como uno de los elementos más influyentes en el desarrollo social (Santrock, 2007). Según Sadurní, Rostán y Serrat (2003) los niños y las niñas inician alrededor de los 3 años una etapa que favorece el desarrollo de las capacidades y habilidades para establecer relaciones sociales. A estas edades se produce un cambio importante dado que los infantes entran en contacto con un nuevo agente socializador: la escuela. Es en este contexto donde el alumnado se enfrenta a la aceptación de nuevas normas de convivencia, a la asunción de nuevos roles y a la interacción con sus iguales. Por tanto, la educación recibida a lo largo de todo el curso académico favorece, como es lógico, la mejora del desarrollo social de la persona. No obstante, teniendo en cuenta los resultados encontrados, se concluye que la intervención directa y sistemática sobre determinados comportamientos sociales a través de un programa de intervención, contribuye de forma evidente al desarrollo social del sujeto, pues acelera y garantiza la adquisición de determinados comportamientos y habilidades de interacción social. El alumnado que ha participado en el programa posee más habilidades para entablar amistades, para mostrarse empático, tiene más capacidad de autocontrol y muestra mayor respeto hacia el seguimiento de las normas.

Los resultados obtenidos en competencia social coinciden con estudios previos que han mostrado los efectos positivos de este programa de intervención, en este caso, con alumnado de 4 años de edad (Benítez et al., 2011a). Del mismo modo, las mejoras reflejadas en el grupo experimental tras la intervención, concuerdan con los resultados hallados recientemente en otros estudios que también evaluaron comportamientos prosociales entre la población infantil (Moraru et al., 2011; Ştefan y Miclea, 2013). Concretamente, el programa PATHS Preschool llevado a cabo con alumnado de 3 años, produjo efectos positivos en variables como la cooperación y la independencia social después de un año de intervención, aunque en este caso los participantes procedían de un entorno socio-económico bajo (Domitrovich et al., 2007).

Por otra parte, los resultados obtenidos en problemas de conducta muestran cómo el alumnado que participa en el programa disminuye de forma significativa todas sus puntuaciones en comparación con el alumnado del grupo control, confirmándose otra de las hipótesis del trabajo (H2). Después de la implementación del programa Aprender a Convivir las niñas y los niños tienen menos niveles de ansiedad y timidez, no se muestran tan tensos o nerviosos, demuestran más afecto e interés por los otros, son más capaces de mantener la atención y manifiestan menos conductas agresivas en el aula, en definitiva, reducen sus problemas de conducta interiorizados y exteriorizados. Los grupos experimental y control han seguido trayectorias distintas a lo largo del periodo de investigación, pues mientras en el grupo experimental se ha observado una reducción significativa en problemas internos y externos, entre los participantes del grupo control no se ha producido ningún cambio destacable. En este sentido, se pone de manifiesto la relación negativa existente entre la competencia social y los problemas de conducta, pues el haber incidido de forma sistemática sobre el desarrollo de la competencia social, ha repercutido en la reducción de problemas de conducta. Estos resultados son congruentes con otros estudios llevados a cabo con población infantil, que también han establecido esta relación entre las variables (Bornstein et al., 2010). Así pues, los niños y niñas que desarrollan y adquieren una competencia social adecuada, es más probable que manifiesten pocos problemas de conducta (interiorizados y exteriorizados), siendo la competencia social un factor de protección frente al desarrollo de problemas como el negativismo (Betina, 2010), la timidez o la ansiedad, además de facilitar la adaptación social, académica y psicológica (Ferrer-Wreder, 2014; Paula, 2001).

Atendiendo de forma más pormenorizada a las variables de interiorización y exteriorización, es destacable señalar que es en la primera donde se producen mayores avances dentro del grupo experimental. En interiorización de problemas los efectos de interacción entre el momento de evaluación y la condición reflejan cómo el grupo experimental manifiesta menos problemas en la fase post que el grupo control. Teniendo en cuenta que dentro de interiorización de problemas se midieron variables como la reactividad emocional, la ansiedad o la timidez, se concluye que el programa Aprender a Convivir contribuye a la mejora del alumnado en estos aspectos. En el programa de intervención se trabajan contenidos relacionados con la comunicación, tratando de que el alumnado aprenda a expresar sus necesidades, sus ideas, sus sentimientos, a decirle cosas bonitas a los compañeros, todo ello teniendo en cuenta unas normas básicas de comunicación social (escuchar, pedir el turno de palabra, etc.). Aprender a Convivir también promueve el aprendizaje de comportamientos prosociales como compartir y ayudar a los compañeros, favorece el trabajo en equipo, y además, incide en el ámbito emocional con el objetivo de que los niños y las niñas apren- 
dan a identificar, expresar y controlar algunas emociones básicas como la alegría o el enfado.

En relación con la exteriorización de problemas el grupo experimental también mejora y marca diferencias con respecto al grupo control después de la intervención. Sin embargo, en el grupo control no se produce ningún cambio e incluso aumenta levemente su puntuación. Dentro de exteriorización, se midieron las variables de comportamiento agresivo y problemas de atención. El programa Aprender a Convivir ha influido en la mejora de estas variables y ello puede deberse a que promueve determinadas estrategias de resolución de problemas y de control de emociones (para-piensa-actúa), que contribuyen al control de la impulsividad. La metodología propia del programa, a través de marionetas, también ayuda a captar la atención del alumnado. Por otro lado, la promoción de determinadas normas como respetar el turno de palabra, pedir permiso para hacer algo, respetar los turnos de juego, atender al maestro o escuchar a los compañeros, también favorecen la autorregulación de la persona.

En definitiva, los resultados alcanzados en la variable de problemas de conducta son muy positivos máxime teniendo en cuenta que el programa de intervención incide directamente sobre la competencia social y no sobre los problemas de conducta. En este sentido, cabe destacar la influencia que el programa Aprender a Convivir ha ejercido sobre esta variable, tal y como señala el tamaño del efecto.

Los resultados obtenidos tanto en competencia social como en problemas de conducta, coinciden con los de otras investigaciones llevadas a cabo con niños/as de edades tempranas que han evaluado el impacto de un programa de intervención sobre la competencia social y los problemas de conducta (Benítez et al., 2011a; Fernández, 2010; Fernández, Benítez, Fernández, Justicia y Justicia-Arráez, 2011; Gunter et al., 2012; Koglin y Petermann, 2011; Lynch, Geller y Schmidt, 2004), e igualmente, están en la línea de algunos estudios de carácter longitudinal (Alba, 2013; Justicia-Arráez, 2014; McKinney, Montague y Hocutt, 1998; Reid, WebsterStratton y Hammond, 2007).

En relación con el sexo, se verifican las últimas hipótesis de la investigación ya que las niñas obtienen puntuaciones más elevadas en las variables de competencia social $(\mathrm{H} 3)$ y puntuaciones más bajas en problemas de conducta exteriorizados (H4) que los niños. Los resultados hallados en competencia social coinciden con los de otros estudios que indican que las niñas muestran mejores niveles que los niños en esta variable (Paterski, Golombok, y Hines, 2011). El metaanálisis elaborado por Fabes y Eisenberg (1998), analizaba las diferencias de sexo en el comportamiento prosocial y encontraron que las niñas mostraban niveles más altos, coincidiendo así con Walker (2005), donde el profesorado señaló diferencias a favor de las niñas entre los 3 y los 5 años en estas mismas variables. También con población infantil de 5-6 años, Öztürk Samur (2014) encontró que las niñas dominaban más habilidades de tipo emocional, como el reconocimiento de emociones. En edades más avanzadas, las diferencias de género también son manifiestas. En este sentido,
Romer, Ravitch, Tom, Merrell y Wesley (2011) hallaron diferencias claras a favor de las niñas (8-10 años) en cuanto a comportamientos empáticos, autorregulación y competencia social. No obstante, estudios similares al presentado aquí, llevados a cabo con sujetos de la misma edad, no encontraron diferencias de género entre los niños y las niñas en competencia social (Moraru et al., 2011; Ştefan y Miclea, 2013).

En cuanto a los problemas de conducta, los niños manifestaron más problemas exteriorizados que las niñas $(\mathrm{H} 4)$ y también más problemas de tipo interiorizado. Este último resultado no se esperaba y contrasta con aquellas investigaciones que señalan que a edades tempranas, los problemas internos se manifiestan de forma similar en ambos sexos (Doey et al., 2013; Offord, et al., 1987). La interiorización de problemas suele ser más prevalente en niñas durante la adolescencia, pero en la infancia los datos son más contradictorios y no existen diferencias tan marcadas (Romer et al., 2011). No obstante, existen estudios transculturales que han evidenciado que los niños con edades comprendidas entre $\operatorname{los} 6$ y los 17 años manifiestan menos problemas internos que las niñas (Crijnen, Achenbach y Verhulst, 1997; Rescorla et al., 2007). Aunque en estos estudios no se incluía población española, los hallazgos coinciden con los de la investigación que nos ocupa, donde los niños de 3 años han puntuado más alto en interiorización de problemas que las niñas. Sería conveniente seguir investigando sobre este tema, sobre todo con niños/as de edades tempranas, pues en la etapa infantil es cuando resulta más difícil detectar diferencias sistemáticas (Shaw, Guilliom, Ingoldsby y Nagin, 2003).

La confirmación de la cuarta hipótesis de la investigación, esto es, que los niños manifiestan más problemas exteriorizados que las niñas, es congruente con los hallazgos obtenidos por otros estudios tanto en población infantil como adolescente (Crijnen et al., 1997; Reid et al., 2000; Rescorla et al., 2007). A pesar de que las diferencias de género en exteriorización de problemas es más consistente, algunos estudios señalan que las diferencias entre ambos sexos pueden diferir según el tipo de conducta evaluada y la edad del sujeto. Investigaciones de revisión meta-analítica han confirmado que en la infancia los niños usan más la agresión física que las niñas (Archer, 2004; Card, Stucki, Sawalani y Little, 2008) mientras que a nivel teórico se ha hipotetizado que las niñas muestran mayores niveles de agresión relacional/encubierta que los niños (Archer y Coyne, 2005). En este sentido, es importante tener en cuenta el instrumento utilizado en esta investigación y tomar con cautela los resultados obtenidos, pues la dimensión de comportamiento agresivo evaluada incluye ítems más vinculados a la agresión física y directa que a una agresión de tipo encubierto.

\section{Conclusiones}

A la luz de los resultados obtenidos, se puede concluir que el programa Aprender a Convivir contribuye a la mejora de la competencia social y a la reducción de los problemas de conducta del alumnado de 3 años, demostrándose que tiene 
mayor efecto sobre estas variables que la mera escolarización. Tales evidencias realzan el papel que tiene la competencia social como factor de protección, pues se demuestra que el desarrollo de la competencia social produce efectos positivos sobre los comportamientos sociales y también sobre los problemas de conducta internos y externos. En este sentido, considerando la influencia que la competencia social tiene sobre los problemas de conducta, la intervención temprana resulta esencial y el programa Aprender a Convivir puede ayudar a frenar la aparición de comportamientos de este tipo, favoreciendo así el ajuste psicológico y el bienestar social del individuo. El aumento de la escolarización en el segundo ciclo de la Educación Infantil y las características propias de esta etapa educativa facilitan la implantación de intervenciones de carácter preventivo. Es importante trabajar la competencia social de una forma sistemática y, en este sentido, el enfoque globalizador que caracteriza la Educación Infantil supone una ventaja para adecuar y encajar contenidos de este campo dentro del currículum. Favorecer este tipo de competencia desde la escuela resulta crucial dado que autores como Monjas (2012), afirman que la falta de competencia social se asocia con una baja aceptación social, problemas escolares y personales, desajustes psicológicos, inadaptación juvenil y problemas futuros de salud mental, además de ser uno de los factores predictores del rendimiento académico (Inglés et al., 2009). Sin embargo, es necesaria una mayor investigación sobre la efectividad de los programas de intervención en estas edades, especialmente para demostrar el papel que tienen

\section{Referencias}

Achenbach, T., y Rescorla, L. A. (2000). Manual of ASEBA preschool forms and profiles. Burlington: University of Vermont, Departament of Psychiatry.

Alba, G. (2013). El programa Aprender a Convivir. La mejora de la competencia social y la reducción de los problemas de conducta en educación infantil y primaria. (Tesis doctoral, publicada). Universidad de Granada, Granada.

Archer, J. (2004). Sex differences in aggression in real-world settings: a meta-analytic review. Review of General Psychology, 8(4), 291-322. doi:10.1037/1089-2680.8.4.291

Archer, J., y Coyne, S. M. (2005). An integrated review of indirect, relational, and social aggression. Personality and Social Psychology Review, 9(3), 212230. doi:10.1207/s15327957pspr0903_2

Alba, G., Justicia-Arráez, A., Pichardo, M. C., y Justicia, F. (2013). Aprender a Convivir. A program for improving social competence in preschool and elementary school children. Electronic Journal of Research in Educational Psychology, 11(3), 843-904. doi: 10.14204/ejrep.31.13105

Benítez, J. L., Fernández, M., Justicia, F., Fernández, E., y Justicia-Arráez, A. (2011a). Results of the Aprender a Convivir program for development of social competence and prevention of antisocial behavior in 4-yearold children. School Psychology International, 32(1), 3-19. doi: 10.1177/0143034310396804

Benítez, J. L., Pichardo, M. C., García, T., Fernández, M., Justicia, F., y Fernández, E. (2011b). Análisis de la estructura factorial de las puntuaciones de la Preschool and Kindergarten Behavior Scale en población española. Psicothema, 23(2), 314-321.

Bergman, L. R., Andershed, H., y Andershed, A. K. (2009). Types and continua in developmental psychopathology: Problem behaviors in school and their relationship to later antisocial behavior. Developmental and Psychopathology, 21, 975-992. doi: 1 0.1017/S0954579409000522

Betina, A. (2010). Las habilidades sociales como recursos para el desarrollo de fortalezas en la infancia. Psicodebate. Psicología, Cultura y Sociedad, 10, 231-248. frente a la mejora debida al desarrollo, utilizando diseños longitudinales que incluyan grupos control (Tolan y Guerra, 1996).

Por otra parte, las diferencias halladas entre niños y niñas de tan corta edad deben ser tenidas en cuenta a la hora de intervenir, con el objetivo de que dicha intervención resulte más efectiva entre la población infantil. El programa fue diseñado para trabajar con toda la población y no se incluyeron propuestas específicas dirigidas a niños o a niñas. En este sentido, también sería conveniente analizar a través de estudios longitudinales, si la implementación de programas que trabajen la competencia social ayudaría a reducir las diferencias entre los niños y las niñas o por lo menos, a minimizarlas.

Agradecimientos.- Esta investigación se ha desarrollado dentro del Proyecto $\mathrm{I}+\mathrm{D}+\mathrm{i}$ Estudio longitudinal de los efectos del programa Aprender a Convivir en el desarrollo de la competencia social y la prevención del comportamiento antisocial (EDU2009-11950), financiado por el Ministerio de Ciencia e Innovación y los Fondos Feder (Fondo Social Europeo). Además, el estudio también ha sido realizado gracias a la concesión de una beca de Formación del Personal Investigador (FPI), financiada por el Ministerio de Ciencia e Innovación (BES2010-030181); y gracias a una beca de Formación del Personal Universitario (FPU), financiada por el Ministerio de Educación y Ciencia (AP2010-2367). También queremos hacer explícito nuestro agradecimiento a los centros educativos que han colaborado en este proyecto.

Bornstein, M. H., Hahn, C. S., y Haynes, O. M. (2010). Social competence, externalizing, and internalizing behavioral adjustment from early childhood through early adolescence: Developmental cascades. Development and Psychopathology, 22(4), 717. doi: 10.1017/S0954579410000416

Bradshaw, C. P., Bottiani, J. H., Osher, D., y Sugai, G. (2014). The integration of positive behavioral interventions and supports and social and emotional learning. En M. D. Weist, N. A. Lever, C. P. Bradshaw y J. S. Owens (Eds.), Handbook of School Mental Health. Research, Training, Practice and Policy (2nd ed., pp. 101-118). New York: Springer. doi:10.1007/9781-4614-7624-5_8

Burt, K. B., Obradović, J., Long, J. D., y Masten, A.S. (2008). The interplay of social competence and psychopathology over 20 years: testing transactional and cascade models. Child Development, 79(2), 359-374. doi: 10.1111/j.1467-8624.2007.01130.x

Calkins, S., y Keane, S. P. (2009). Developmental origins of early antisocial behavior. Developmental and Psychopathology, 21, 1095-1109. doi:10.1017/S095457940999006X

Card, N. A., Stucky, B. D., Sawalani, G. M., y Little, T. D. (2008). Direct and indirect aggression during childhood and adolescence: a metaanalytic review of gender differences, intercorrelations, and relations to maladjustment. Child Development, 79(5), 1185-1229. doi:10.1111/j.14678624.2008.01184.x

Cohen, J. (1988). Statistical power analysis for the behavioral sciences (2nd edition). Hillsdale, NJ: Erlbaum

Cole, D. A., Martin, J. M., Powers, B., y Truglio, R. (1996). Modeling causal relations between academic and social competence and depression: a multitrait-multimethod longitudinal study of children. Journal of Abnormal Psychology, 105(2), 258-270.

Cooper, J., Goodfellow, H., Muhlheim, E., Paske, K., y Pearson, L. (2003). PALS social skills program: Playing And Learning to Socialise: Resource Book. Inscript Publishing. 
Crijnen, A. A., Achenbach, T. M., y Verhulst, F. C. (1997). Comparisons of problems reported by parents of children in 12 cultures: Total problems, externalizing and internalizing. Journal of the American Academy of Child and Adolescent Psychiatry, 36, 1269-1277.

Daly, B. P., Nicholls, E., Aggarwal, R., y Sander, M. (2014). Promoting social competence and reducing behavior problems in at-risk students: implementation and efficacy of universal and selective prevention programs in schools. En M. D. Weist, N. A. Lever, C. P. Bradshaw y J. S. Owens (Eds.), Handbook of school mental health. Research, training, practice and policy (2nd ed., pp. 131-144). New York: Springer.

Del Prette, Z. A. P., Del Prette, A., De Oliveira, L. A., Gresham, F. M., y Vance, M. J. (2012). Role of social performance in predicting learning problems: Prediction of risk using logistic regression analysis. School Psychology International, 33(6), 615-630. doi:10.1177/0020715211430373

Dodge, K. A., Godwin, J., y The Conduct Problems Prevention Research Group. (2013). Social-information-processing patterns mediate the impact of preventive intervention on adolescent antisocial behavior. Psychological Science, 24(4), 456-465. doi: 10.1177/0956797612457394

Doey, L., Coplan, R. J., y Kingsbury, M. (2013). Bashful boys and coy girls: a review of gender differences in childhood shyness. Sex Roles, 70(7-8), 255-266. doi:10.1007/s11199-013-0317-9

Domitrovich, C. E., Cortes, R. C., y Greenberg, M. T. (2007). Improving young children's social and emotional competence: a randomized trial of the preschool "PATHS" curriculum. The Journal of Primary Prevention, 28(2), 67-91. doi:10.1007/s10935-007-0081-0

Domitrovich, C., Greenberg, M., Kusche, C., y Cortes R. (2004). PATHS preschool program. South Deerfield, MA: Channing Bete Company.

Eisenberg, N. (2000). Emotion, regulation, and moral development. Annual Review Psychology, 51, 665-697. doi: 10.1146/annurev.psych.51.1.665

Eron, L. D. (1990). Understanding aggression. Bulletin of the International Society for Research on Aggression, 12, 5-9.

Fabes, R. A., y Eisenberg, N. (1998). Prosocial development. En W. Damon (Ed.), Handbook of Child Psyhology (5 $5^{\text {th }}$ ed.). New York: J. Wiley.

Fernández, M. (2010). Desarrollo de la competencia social en niños de tres años como prevención de problemas de conducta. (Tesis doctoral). Universidad de Granada, Granada.

Fernández, M., Benítez, J. L., Fernández, E., Justicia, F. y Justicia-Arráez, A. (2011). Desarrollo de la competencia social y prevención del comportamiento antisocial en niños de 3 años. Infancia y aprendizaje, 34(3), 337347. doi: 10.1174/021037011797238603

Fernández, M., Benítez, J. L., Pichardo, M. C., Fernández, E., Justicia, F., García, T., ... Alba, G. (2010). Análisis factorial confirmatorio de las subescalas del PKBS-2, para la evaluación de las habilidades sociales y los problemas de conducta, en educación infantil. Electronic Journal of Research in Educational Psychology, 8(22), 1229-1252.

Ferrer-Wreder, L. (2014). Advancing child and adolescente well-being through positive youth development and prevention programs. En A. Ben Arieh, F. Casas, I. Frønes, y J. E. Korbin, (Eds.). (2014). Handbook of child well-being. Theories, methods and policies in global perspectives. Vol. 2. (pp. 3025-3041). Springer-Verlag. doi: 10.1007/978-90-481-9063-8_164

Gunter, L., Caldarella, P., Korth, B. B., y Young, K. R. (2012). Promoting social and emotional learning in preschool students: a study of Strong Start Pre-K. Early Childhood Education Journal, 40(3), 151-159. doi:10.1007/s10643-012-0507-z

Hemphill, S. A. (1996). Characteristics of conduct-disordered children and their families: a review. Australian Psychologist, 31(2), 109-118. doi:10.1080/00050069608260189

Inglés, C., Benavides, G., Redondo, J., García-Fernández, J. M., RuizEsteban, C., Estévez, C., y Huescar, E. (2009). Conducta prosocial y rendimiento académico en estudiantes españoles de Educación Secundaria Obligatoria. Anales de psicología, 25(1), 93-101.

Justicia, F., Benítez, J. L., Fernández, M., Fernández, E., y Pichardo, M. C. (2008). Aprender a convivir: programa de prevención do comportamento antisocial na educación infantil. Cadernos de psicoloxía, 32, 37- 47.

Justicia-Arráez, A. (2014). Estudio longitudinal de los efectos del programa Aprender a Convivir en Educación Infantil. (Tesis doctoral, publicada). Universidad de Granada, Granada.

Joseph, G. E., y Strain, P. S. (2003). Comprehensive evidence-based socialemotional curricula for young children: an analysis of efficacious adop- tion potential. Topics in Early Childhood Special Education, 23(2), 62-73. doi: $10.1177 / 02711214030230020201$

Keenan, K., y Shaw, D. (1994). The development of aggression in toddlers: A study of low income families. Journal of Abnormal Child Psychology, 22(1), 53-78. doi: 10.1007/bf02169256

Koglin, U., y Petermann, F. (2011). The effectiveness of the behavioural training for preschool children. European Early Childhood Education Research Journal, 19(1), 97-111. doi:10.1080/1350293X.2011.548949

Kramer, T. J., Caldarella, P., Christensen, L., y Shatzer, R. H. (2010). Social and emotional learning in the kindergarten classroom: evaluation of the Strong Start curriculum. Early Childhood Education Journal, 37(4), 303309. doi:10.1007/s10643-009-0354-8

Larmar, S., Dadde, M. R., y Shochet, I. (2006). Successes and challenges in preventing conduct problems in Australian preschool-aged children through the Early Impact (EI) Program. Behaviour Change, 23(2), 121137. doi: $10.1375 /$ bech.23.2.121

Lynch, K. B., Geller, S. R., y Schmidt, M. G. (2004). Multi-year evaluation of the effectiveness of a resilience-based prevention program for young children. The Journal of Primary Prevention, 24(3), 335-353. doi: 10.1023/B:JOPP.0000018052.12488.d1

McKinney, J. D., Montague, M., y Hocutt, A. M. (1998). Systematic screening of children at risk for developing SED: initial results from a prevention project. En D. J. Liberton, K. Kutash y R. M. Friedman (Eds.). The $10^{\text {th }}$ annual research conference proceedings, a system of care for children's mental health: expanding the research base (pp. 271-276). Tampa: University of South Florida.

Merrell, K.W. (2002). Preschool and Kindergarten Behavior Scales. Austin, TX: Pro-ed.

Mesman, J., Bongers, I. J., y Koot, H. M. (2001). Preschool developmental pathways to preadolescent internalizing and externalizing problems. Journal of Child Psychology and Psychiatry, 42(5), 679-689. doi: 10.1111/1469-7610.00763

Monjas, M. I. (2012). Programa de enseñanza de habilidades de interacción social (PEHIS) para niños y niñas en edad escolar $\left(10^{\mathrm{a}} \mathrm{ed}\right.$. Rev.). Madrid: CEPE.

Monjas, M. I. (Dir.). (2011). Cómo promover la convivencia: Programa de Asertividad y Habilidades Sociales (PAHS). Educación Infantil, Primaria y Secundaria ( $3^{\mathrm{a}}$ ed.) Madrid: Editorial CEPE.

Monjas, M. I., y González, B. (1998). Las habilidades sociales en el currículo. España. Ministerio de Educación, Cultura y Deporte. Centro de Investigación y Documentación Educativa. (CIDE). 146. Colección: Investigación.

Moraru, A., Stoica, M., Tomuletiu, E. A., y Filpisan, M. (2011). Evaluation of a program for developing socio-emotional competencies in preschool children. Procedia-Social and Behavioral Sciences, 30, 2161-2164. doi: 10.1016/j.sbspro.2011.10.419

Obradović, J., Burt, K. B., y Masten, A. S. (2009). Testing a dual cascade model linking competence and symptoms over 20 years from childhood to adulthood. Journal of Clinical Child \& Adolescent Psychology, 39(1), 90-102. doi:10.1080/15374410903401120

Offord, D. R., Boyle, M. H., Szatmari, P., Rae-Grant, N. I., Links, P. S., Cadman, D. T.,... y Woodward, C. A (1987). Ontario child health study: II. six-month prevalence of disorder and rates of service utilization. Archives of General Psychiatry, 44, 832-836. doi: 10.1001/archpsyc.1987.01800210084013

Öztürk Samur, A. (2014). A study on the relationship between externalising behaviours and emotional skills of 60-72-month-old children. Early Child Development and Care, 1-9. doi:10.1080/03004430.2014.905549

Paterski, V., Golombok, S., y Hines, M. (2011). Sex differences in social behavior. En P. K. Smith y C. H. Hart, The Wiley-Blackwell Handbook of Childhood Social Development, (2a ed., pp. 283-412). Chicheser (UK): Wiley Blackwell. doi: 10.1002/9781444390933.ch15

Paula, I. (2001). Competencia social y adaptación social y psicológica. Aula de Innovación Educativa, 102, 13-17.

Petermann, F., y Natzke, H. (2008). Preliminary results of a comprehensive approach to prevent antisocial behavior in preschool and primary school pupils in Luxembourg. School Psychology International 29(5), 606626. doi: 10.1177/0143034308099204

Reid, R., Riccio, C. A., Kessler, R. H., DuPual, G. J., Power, T. J., Anastopoulos, A. D., Rogers-Adkinson, D., y Nol, M. B. (2000). Gender and ethnic differences in ADHD as assessed by behavior ratings. Journal of 
Emotional and Behavioral Disorders, 8, 38-48. doi: $10.1177 / 106342660000800105$

Reid, M. J., Webster-Stratton, C., y Hammond, M. (2007). Enhancing a classroom social competence and problem-Solving curriculum by offering parent training to families of moderate- to high risk elementary school children. Journal of Clinical Child and Adolescent Psychology, 36(4), 605-620. doi: 10.1080/15374410701662741

Rescorla, L., Achenbach, T., Ivanova, M. Y., Dumenci, L., Almqvist, F., Bilenberg, N., ... y Verhulst, F. (2007). Behavioral and emotional problems reported by parents of children ages 6 to 16 in 31 societies. Journal of Emotional and Behavioral Disorders, 15(3), 130-142. doi:10.1177/10634266070150030101

Reynolds, A. J., y Temple, J. A. (2008). Cost-effective early childhood development programs from preschool to third grade. Annual Review of $\begin{array}{llll}\text { Clinical Psychology, } & 4(1), & 109-139 .\end{array}$ doi:10.1146/annurev.clinpsy.3.022806.091411

Reynolds, A. J., y Temple, J. A. (2012). Economic returns of investments in preschool education. En E. Zigler, W. S. Guilliam y S. M. Jones, $A$ visión for universal preschool education, (pp. 37-68). [Cambridge Books Online]. doi: 10.1017/CBO9781139167284.004 (Trabajo original publicado en 2006).

Reynolds, A. J., Temple, J. A., Ou, S. R., Robertson, D. L., Mersky, J. P., Topitzes, J. W., y Niles, M. D. (2007). Effects of a school-based, early childhood intervention on adult health and well-being. Archives of Pediatrics \& Adolescent Medicine, 161(8), 730-739. doi:10.1001/archpedi.161.8.730

Romer, N., Ravitch, N. K., Tom, K., Merrell, K. W., y Wesley, K. L. (2011). Gender differences in positive social-emotional functioning. Psychology in the Schools, 48(10), 958-970. doi: 10.1002/pits.20604

Rose-Krasnor, L. (1997). The nature of social competence. A theoretical review. Social Development, 6(1), 111-135. doi: 10.1111/j.14679507.1997.tb00097.x

Sadurní, M., Rostán, C., y Serrat, C. (2003). El desarrollo de los niños paso a paso. Barcelona: UOC

Santrock, J. (2007). Desarrollo Infantil. México: McGraw-Hill.

Shaw, D. S., Gilliom, M., Ingoldsby, E. M., y Nagin, D. S. (2003). Trajectories leading to school-age conduct problems. Developmental Psychology, 39(2), 189-200. doi:10.1037/0012-1649.39.2.189
Schulte-Rüther, M., Markowitsch, H. J., Shah, N. J., Fink, G. R., y Piefke, M. (2008). Gender differences in brain networks supporting empathy. Neuroimage, 42(1), 393-403. doi:10.1016/j.neuroimage.2008.04.180

Snyder, H. (2001). Epidemiology of official offending. En R. Loeber y D. P. Farrington (Eds.), Child delinquents: Development, intervention and service needs (pp.25-46). Thousand Oaks, CA: Sage.

Sørlie, M. A., Hagen, K. A., y Ogden, T. (2008). Social competence and antisocial behavior: Continuity and distinctiveness across early adolescence. Journal of Research on Adolescence, 18(1), 121-144. doi: $10.1111 /$ j. $1532-7795.2008 .00553 . x$

Ştefan, C. A., y Miclea, M. (2013). Effects of a multifocused prevention program on preschool children's competencies and behavior problems. Psychology in the Schools, 50, 382-402. doi:10.1002/pits.21683.

Tolan, R. H., y Guerra, N. G. (1996). Progress and prospects in youth violence-prevention evaluation. American Journal of Preventive Medicine, 12, $129-131$.

Tremblay, R. E. (2007). The development of youth violence: an old story with new data. European Journal on Criminal Policy and Research, 13(3-4), 161-170. doi:10.1007/s10610-007-9050-7

Vaughn, B. E., Shin, N., Kim, M., Coppola, G., Krzysik, L., Santos, A. J., ... y Korth, B. (2009). Hierarchical models of social competence in preschool children: a multisite, multinational study. Child development, 80(6), 1775-1796. doi: 10.1111/j.1467-8624.2009.01367.x

Walker, S. (2005). Gender differences in the relationship between young children's peer-related social competence and individual differences in theory of mind. The Journal of Genetic Psychology, 166(3), 297-312. doi 10.3200/GNTP.166.3.297-312

Waters, E., y Sroufe, L. A. (1983). Social competence as a developmental construct. Developmental review, 3(1), 79-97. doi: 10.1016/02732297(83)90010-2

Zins, J., Weissberg, R., Wang, M. C., y Walberg, H. J. (2004). Building academic success on social and emotional learnig (SEL): What does the research say? New York: Teachers College Press.

(Articulo recibido: 11-11-2013; revisado: 08-12-2014; aceptado: 17-12-2014 